\title{
Organização e qualidade da assistência à saúde dos índios Kaingáng do Rio Grande do Sul, Brasil
}

\author{
Organization and quality of health care for \\ Kaingáng Indians in Rio Grande do Sul, Brazil
}

Yara Hahr Marques Hökerberg 1

Milena Piraccini Duchiade 2

Christovam Barcellos 3

\footnotetext{
1 Hospital Municipal Souza Aguiar, Secretaria Municipal de Saúde do Rio de Janeiro. Praça da República 111, Rio de Janeiro, $R J$ 20211-350, Brasil. 2 Escola Nacional de Saúde Pública, Fundação Oswaldo Cruz. Rua Leopoldo Bulhões 1480, Rio de Janeiro, RJ 21041-210, Brasil.

3 Departamento de Informações em Saúde, Centro de Informação Científica e Tecnológica, Fundação Oswaldo Cruz. Av. Brasil 4365, Rio de Janeiro, $R J$ 21045-900, Brasil.
}

\begin{abstract}
This study assesses the health care provided to Kaingáng Indians in Rio Grande do Sul, Brazil. Deaths preventable by primary health care among the Indians and occurring from 1985 to 1995 were compared to the same rates for the State of Rio Grande do Sul as a whole. Secondary data on health care services were supplemented by field interviews with indigenous leaders and with employees from participating institutions. A Geographic Information System (GIS) was used to correlate distribution of deaths and access to health services. The Kaingáng settlements are connected by paved roads to counties with at least a public health clinic or even a small hospital in some cases. Secondary referrals are treated in Palmeiras das Missões and Frederico Westphallen and tertiary care is provided in Passo Fundo. What distinguishes the Indian settlements from the rest of the State are the high rates of deaths preventable by primary health care and those related to ill-defined conditions, malnutrition, tuberculosis, and cancer of the uterine cervix.
\end{abstract}

Key words Health Status Indicators; Information System; Kaingáng; South American Indians

Resumo Este estudo pretende avaliar o atendimento à saúde prestado aos índios Kaingáng do Rio Grande do Sul. Os óbitos evitáveis por assistência básica de saúde dos indígenas, ocorridos entre 1985 e 1995, foram comparados aos óbitos do Estado do Rio Grande do Sul. Os dados secundários relativos aos serviços de saúde foram complementados por entrevistas realizadas em campo com lideranças indígenas e com funcionários das instituições envolvidas na assistência à saúde do índio. Foi utilizado o Sistema de Informações Geográficas, visando relacionar a distribuição dos óbitos ao grau de acesso aos serviços de saúde. As áreas indígenas Kaingáng estão ligadas por estradas pavimentadas a municípios que contam com, pelo menos, um centro de saúde, ou ainda, hospitais de pequeno porte. O segundo nível de atenção situa-se em Palmeiras das Missões e Frederico Westphallen e o terceiro nível, em Passo Fundo. O que diferencia estas áreas indígenas do restante do Estado do Rio Grande do Sul é a quantidade de óbitos evitáveis pela assistência básica de saúde, tais como as causas mal definidas, a desnutrição, a tuberculose e o câncer cérvico-uterino.

Palavras-chave Indicadores de Saúde; Sistema de Informação; Kaingáng; Índios Sul-Americanos 


\section{Introdução}

Este trabalho propõe-se a analisar a situação de saúde vivida pelos índios Kaingáng, relacionando-a com seu acesso aos serviços de saúde. Foi realizada uma análise descritiva dos dados disponíveis, avaliando-se a qualidade dos mesmos e complementando-os com entrevistas qualitativas. Nosso objetivo foi o de subsidiar políticas no campo da saúde do índio, orientadas, mais especificamente, aos índios Kaingáng do Rio Grande do Sul.

A análise da situação de saúde corresponde a uma das etapas do processo de tomada de decisão na planificação, o que inclui ainda a definição do problema, o estabelecimento de metas e objetivos e, por último, a elaboração de um plano de operações, bem como dos indicadores para avaliar os resultados, com a intenção de mobilizar ações viáveis de transformação da situação anterior (Castellanos, 1990; White, 1998).

Para Donabedian (1966), o fator primordial para avaliar a qualidade da atenção em saúde é a proteção, a produção e a satisfação em saúde. Apesar da relevância do tema é notório o escasso número de publicações disponíveis a esse respeito, além do parco desenvolvimento conceitual e metodológico nelas evidenciado (OPS, 1988).

A questão indígena é marcada, de modo geral, pela dispersão de pequenos grupos de população, pela privação de terras e conseqüentes disputas pela demarcação das mesmas ou ampliação das áreas anteriormente demarcadas, pelas mudanças ecológicas e culturais, que não se traduzem em melhoria e, muito menos, na adaptação aos hábitos culturais da sociedade nacional. A deterioração da qualidade de vida resultante do contato com a sociedade envolvente trouxe conseqüências quase sempre dramáticas, caracterizadas por epidemias e fome (Wirsing, 1985), situação que vem perdurando até os dias atuais para os grupos sobreviventes, apesar de variar, em maior ou menor grau, de acordo com as características culturais de cada grupo e as variáveis ecológicas, políticas e socioeconômicas que os rodeiam. Do mesmo modo, o acesso aos serviços de saúde é influenciado por tais características, além do componente distância geográfica (Kunitz, 1983; Young, 1988).

Para Confalonieri (1993), a falta de recursos e a instabilidade político-administrativa das instituições públicas responsáveis pela assistência aos povos indígenas brasileiros geram descontinuidade das ações em saúde. Também, o relacionamento entre os agentes prestadores de serviços de saúde, as instituições públicas e os indígenas não considera, com freqüência, os valores tradicionais da cultura, dificultando a atuação e o estabelecimento de certos diagnósticos. O monitoramento da situação de saúde dos povos indígenas deve ter, como objetivo, a adequação dos serviços às especificidades epidemiológicas, geopolíticas e culturais.

Ainda que os dados epidemiológicos existentes para as áreas indígenas brasileiras sejam pouco precisos e dispersos, a existência destes, por si só, justifica o seu uso em uma análise crítica. Olhar os números como indicadores que apontam um caminho, abandonando a perspectiva estática e atemporal para confrontá-los com as teorias existentes e com os dados da realidade histórica, social e cultural pode ser a saída (De Paula, 1991). Metodologias alternativas têm sido apresentadas, como, por exemplo, o uso do conceito de morte evitável (OPS, 1988), de evento-sentinela (Rutstein, 1976), além de propostas como a apresentada por Lobo (1996), que viabiliza a elaboração de um Sistema de Vigilância Epidemiológica para a área indígena Yanomámi no Estado de Roraima.

Os Sistemas de Informação Geográfica (SIG) são estruturas de processamento eletrônico de dados que permitem a captura, armazenamento, manipulação e análise de dados referenciados geograficamente (OPS, 1996), os quais vêm sendo incorporados ao campo da Saúde Pública para melhorar as possibilidades de descrição e para permitir uma análise espacial das doenças em grandes conjuntos de dados georreferenciados (Barcellos \& Bastos, 1996).

Do ponto de vista epidemiológico e de planejamento em saúde, os SIGs podem auxiliar na compreensão das diferenças na dinâmica espacial do processo saúde-doença, na eleição de prioridades de atuação, na integração interinstitucional e na definição de distritos sanitários especiais indígenas. A agilidade na atualização das informações obtidas pode servir para facilitar o diálogo com as comunidades indígenas pelas possibilidades visuais do sistema, propiciando uma participação mais efetiva na tomada de decisões (OPS, 1996).

Em 1994, os Kaingáng representavam 9.888 índios, distribuídos em onze áreas indígenas localizadas na região noroeste do Rio Grande do Sul (Figura 1) e interligadas por rodovias pavimentadas - tais como a RS-386 e a BR-285 -, circundadas por municípios com realidades socioculturais bem distintas. Sobreviviam de pequenas lavouras de subsistência e do artesanato. A persistência da prática do arrendamento das terras indígenas para colonos da região em Cacique Doble, Guarita, Ligeiro e Nonoai - 


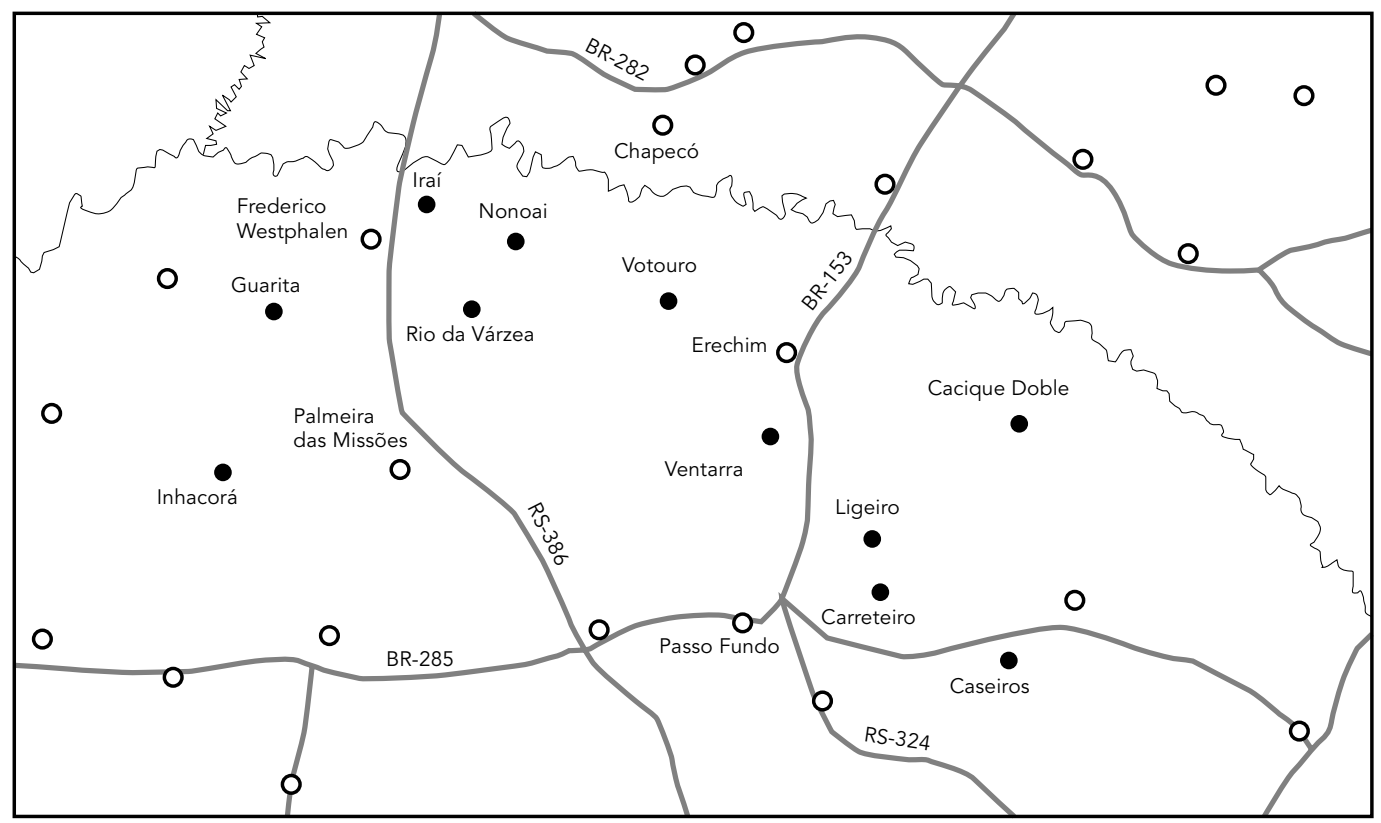

O Principais cidades

- Aldeias Kaigang

Rodovias

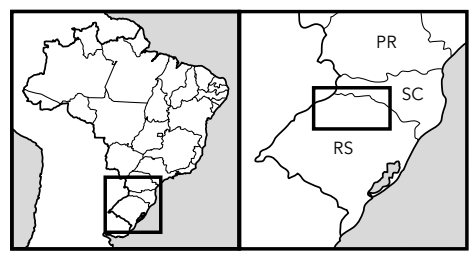

incentivada historicamente pelo Serviço de Proteção ao Índio e, mais recentemente pela Fundação Nacional do Índio (FUNAI) - era o fator que as diferenciava das demais. Atualmente, esta prática vem sendo reprimida pela própria FUNAI, com o auxílio da Polícia Federal, sob a alegação de impedir o desgaste das terras, já intenso, seja pelo desmatamento seja pelo uso indiscriminado de agrotóxicos. Também, o arrendamento favorecia apenas pequena parcela da população indígena, o que se resumia às lideranças, aumentando ainda mais as tensões políticas locais. A situação fundiária não estava resolvida nas áreas de Rio da Várzea, Caseiros e Ventarra, onde as terras ainda não estavam demarcadas. Iraí, apesar de demarcada, possuía um aeroporto no único espaço possível para o plantio. As áreas de Cacique Doble, Guarita, Votouro e Nonoai convivem, de modo conflitante, com índios da etnia Guaraní-Mbyá.
O presente estudo pretende analisar criticamente os dados de mortalidade dos índios Kaingáng do Rio Grande do Sul no período entre 1985 e 1995 e confrontá-los com a organização do atendimento à saúde do índio no referido Estado, em distribuição espacial que permita visualizar o fluxo dos índios em busca de serviços de saúde.

\section{Material e métodos}

Os dados epidemiológicos dos índios Kaingáng foram obtidos através dos relatórios anuais de óbitos de 1985 a 1995 da FUNAI. Foram também utilizados o Sistema de Informação sobre Mortalidade (MS, 1997) para avaliar os óbitos ocorridos durante o ano de 1991 no conjunto da população do Estado do Rio Grande do Sul. 
Informações a respeito da organização e do acesso aos serviços de saúde foram obtidas mediante entrevistas realizadas em campo com as lideranças indígenas, com as mães e com os profissionais de saúde que atuavam, em 1996, na Administração Regional (ADR) da FUNAI em Passo Fundo, na Equipe de Saúde do Índio da Fundação Nacional de Saúde (ESAI/FUNASA/ RS), nas Secretarias Municipais e Estaduais de Saúde. A distribuição de serviços de saúde nos Municípios do Rio Grande do Sul foi obtida através da pesquisa sobre Assistência MédicoSanitária (IBGE, 1992) e estratificados de acordo com a capacidade de atendimento, segundo os critérios de Viacava \& Bahia (1996).

As entrevistas foram efetuadas por intermédio de questionários abertos, dirigidos para resgatar a impressão acerca da qualidade do atendimento prestado e dos serviços de saúde utilizados como referência local, regional e estadual. Foram visitadas as áreas de Ligeiro, Iraí, Rio da Várzea e Guarita (Hökerberg, 1997). A análise das entrevistas baseou-se nos pressupostos da pesquisa qualitativa (Minayo, 1993).

Como indicadores de saúde de interesse foram empregadas a proporção de óbitos evitáveis (OPS, 1988) através de ações preventivas e terapêuticas dos serviços de saúde, aqueles por causas mal definidas e por causas relacionadas ao período perinatal, pneumonias, desnutrição, câncer de colo uterino e tuberculose. Essas condições foram selecionadas a partir de uma lista de uso imediato proposta por Rutstein (1976) (Tabela 1).

O conjunto de dados considerados incluiu a distribuição dos óbitos evitáveis nas áreas indígenas no período entre 1985 e 1995, o fluxo seguido pelos índios para a utilização dos serviços de saúde, a distribuição dos serviços de saúde por nível de complexidade e a distribuição proporcional dos óbitos evitáveis da população não-indígena. A base cartográfica incluiu a malha municipal do estado do Rio Grande do Sul (IBGE, 1997), a localização aproximada das áreas indígenas Kaingáng obtida na FUNAI em 1995 e a divisão do Estado do Rio Grande do Sul em Delegacias Regionais de Saúde (SSMA/ RS, 1993). Esses dados foram integrados em uma base georreferenciada pelos códigos dos municípios de referência para a prestação de serviços de saúde das áreas indígenas.

A análise está baseada em abordagem descritiva que evidencia dois momentos: o primeiro, com a descrição da organização da assistência à saúde do índio no Rio Grande do Sul e o segundo, com a análise espacial do fluxo percorrido pelos índios em busca de atendimento, confrontados com os óbitos evitáveis por assis- tência básica de saúde (Rutstein, 1976). As áreas indígenas de Nonoai, Caseiros e Ventarra foram excluídas dessa etapa da análise, por não terem dados epidemiológicos disponíveis para o período entre 1985 e 1995.

\section{As atribuições e as realizações das instituições responsáveis pela saúde do índio no Rio Grande do Sul: a dicotomia FUNAI-FUNASA}

Historicamente, as ações em saúde das unidades da FUNAI costumavam restringir-se às ações de imunização e à prestação de cuidados primários em saúde. De acordo com o depoimento de um funcionário da FUNAI, o atendimento à saúde do índio durante os últimos quinze anos, no Rio Grande do Sul, poderia ser dividido em dois períodos. O primeiro período, de 1987 a 1990, antes da municipalização da maioria dos municípios de referência para as áreas indígenas, quando todas as atribuições relativas à saúde do índio eram de responsabilidade da FUNAI, em Passo Fundo, e incluíam atividades como a distribuição de medicamentos, o encaminhamento dos pacientes para consultas especializadas ou para internação, o transporte do doente, além das atividades de apoio aos doentes e às suas famílias em Passo Fundo. As referências utilizadas para casos mais graves eram Porto Alegre e Curitiba. O segundo período, após 1991, com a implantação do SUS, caracterizou-se pela maior participação e integração dos municípios com os órgãos responsáveis pela saúde do índio, o que facilitou o acesso aos serviços de saúde. As atividades desenvolvidas na ADR de Passo Fundo (FUNAI) passaram a concentrar-se no encaminhamento dos pacientes, na marcação de consultas e no acompanhamento dos doentes internados (Hökerberg, 1997).

A formação dos recursos humanos para atuar em campo era deficiente. A maioria dos funcionários pertenciam à FUNAI e tinham apenas o primeiro grau; após um treinamento inicial sobre saúde eram qualificados como "atendentes de enfermagem" e passavam a atuar sem supervisão, sem reciclagem, sem referência estruturada e sob forte pressão da comunidade indígena.

A Equipe de Saúde do Índio (ESAI/FUNASA/ RS), localizada em Porto Alegre, iniciou suas atividades após 1992, atendendo ao Decreto Presidencial no 23 de 4/2/1991, que repassou a responsabilidade concernente à saúde do índio da FUNAI para o âmbito da FUNASA. Foram realizados encontros do Núcleo Interinsti- 
tucional de Saúde do Índio (NISI) e treinamentos de saúde. Porto Alegre passou a ser mais utilizada como referência para a atenção terciária.

Em 1994, com o Decreto Presidencial no 1141 de 19/5/1994, a atenção à saúde do índio voltou a ser atribuição da FUNAI e as ações foram redistribuídas. A FUNASA assumiu as responsabilidades quanto às ações de imunização, saneamento, vigilância epidemiológica e treinamento de recursos humanos, enquanto a FUNAI ficou responsável pela assistência à saúde. Os municípios da região atuavam na assistência médica, participavam da imunização e da execução das ações relacionadas aos programas de saúde e a Secretaria de Saúde e Meio Ambiente do Rio Grande do Sul (SSMA/RS) atuava na coordenação dos Programas de tuberculose, hanseníase e no Programa de Agentes Comunitários de Saúde.

No ano de 1996, a equipe da ESAI/FUNASA/ RS, que vinha logrando alguns ganhos no tocante à aproximação com os índios e com as instituições atuantes, foi desmontada. Nesse ano, nada do planejado junto ao NISI foi realizado e, segundo informações dos funcionários da FUNASA, este órgão consumiu apenas $29 \%$ do orçamento anual. Além da construção de poucos módulos sanitários e da proteção de algumas fontes de água, foram distribuídos multimistura e leite para os desnutridos e posta em prática a vacinação na área Guarani M’bya, outra etnia presente na região. Realizaram, ainda, um treinamento para agentes de saúde indígenas aberto aos "atendentes de enfermagem" da FUNAI, com o apoio da organização não governamental Conselho de Missão entre Índios (COMIN), da Igreja Evangélica de Confissão Luterana no Brasil, sediada em Frederico Westphallen (Hökerberg, 1997).

\section{A oferta de serviços de saúde}

A SSMA/RS dividiu operacionalmente o Estado em dezesseis Delegacias Regionais de Saúde (DRS) responsáveis pelo repasse de insumos destinados às atividades do programa de imunização, de tuberculose, de prevenção ao câncer cérvico-uterino e de pré-natal, além da consolidação de informações de interesse epidemiológico. As áreas Carreteiro, Cacique Doble, Caseiros e Ligeiro estavam situadas na $6 \underline{a}$ DRS, cuja sede se localizava em Passo Fundo; Inhacorá, na 9a DRS, com sede em Cruz Alta; Votouro e Ventarra ficavam na 11a DRS, com sede em Erexim; e Guarita, Nonoai, Rio da Várzea e Iraí situavam-se na 15a DRS, cuja sede se localizava em Palmeiras das Missões.
Tabela 1

Enfermidades que produzem óbitos evitáveis por ações preventivas e terapêuticas dos serviços de saúde: índices de uso imediato'.

\begin{tabular}{|c|c|c|}
\hline CID 9ạ revisão² & Enfermidades & Óbito evitável por \\
\hline 010-018 & Tuberculose (todas as formas) & Tratamento \\
\hline 032 & Difteria & Prevenção \\
\hline 033 & Coqueluche & Prevenção \\
\hline 037 & Tétano & Prevenção \\
\hline 045-049 & Poliomielite & Prevenção \\
\hline 055 & Sarampo & Prevenção \\
\hline 060 & Febre Amarela & Prevenção \\
\hline 090 & Sífilis Congênita & Prevenção e Tratamento \\
\hline 091 & Sífilis Primária & Tratamento \\
\hline 098 & Infecção Gonocócica & Tratamento \\
\hline 127 & Ascaridíase & Prevenção e Tratamento \\
\hline 180 & $\begin{array}{l}\text { Neoplasma maligno } \\
\text { de colo uterino }\end{array}$ & Tratamento 3 \\
\hline $260-269$ & Desnutrição & Prevenção e Tratamento \\
\hline 480-487, 490M & Pneumonias e Gripes & Tratamento \\
\hline $760-778$ & Causas Perinatais & Prevenção \\
\hline 780-789, 799 & Sinais e Sintomas Mal Definidos & - \\
\hline
\end{tabular}

1 Adaptação da Lista de uso imediato (Rutstein, 1976).

20 Código Internacional de Doenças (CID) foi adequado à nona revisão.

3 Óbito evitável por diagnóstico e tratamento precoce.

A distribuição da população e dos serviços de saúde nas DRS não era homogênea. Em 1993, estimava-se que $31,5 \%$ da população de todo o Estado concentrava-se na 1a DRS, cuja sede é Porto Alegre, enquanto a 11a DRS abrigava apenas $2,5 \%$, uma das menores parcelas da população gaúcha. A 9ạ DRS abrigava 3,34\%, a 15 a DRS, $4,4 \%$ e a $6 \underline{\text { a DRS }}$ com $5,5 \%$ da população total do Estado. Portanto, as delegacias que englobavam as áreas indígenas Kaingáng se situavam nas áreas menos populosas do Estado do Rio Grande do Sul.

Em relação à oferta de serviços de saúde em 1993 observou-se a seguinte distribuição: enquanto a 1a DRS, que concentrava $13,7 \%$ da população do Estado, tinha 308 unidades ambulatoriais e 48 hospitais, a 6 a DRS, com 3,4\% da população do Rio Grande do Sul e onde se localizava a sede da ADR de Passo Fundo, possuía 191 unidades ambulatoriais e 31 hospitais. A 15 DRS abrigava $1,2 \%$ da população estadual e contava com 189 unidades e 38 hospitais, a 9ạ DRS, com $1 \%$ da população do Rio Grande do Sul, tinha cerca de 123 ambulatórios e 23 hospitais, enquanto a 11 a abrigava $0,2 \%$ da população e possuía 92 ambulatórios e 21 hospitais, correspondendo a uma das localidades com menor concentração de serviços de saúde no Estado, perdendo para a 8ạ e a 7ạ DRS (SSMA/RS, 1993). 
De acordo com informações obtidas na FUNAI e confirmadas em área, o primeiro atendimento prestado ao indígena com problemas de saúde ocorria, em geral, nos postos da FUNAI existentes nas áreas e era feita por "atendentes de enfermagem”, passando a ser, mais recentemente, por auxiliares de enfermagem treinados. Esse atendimento constava de uma triagem que definia quais os pacientes que seriam encaminhados ao atendimento médico. Diferentes caminhos eram percorridos, de acordo com a procedência do paciente e da complexidade do caso.

A rotina de atendimento dos profissionais nos postos indígenas deveria restringir-se à triagem dos pacientes. Porém, esses profissionais acabavam por decidir o tratamento e avaliar a evolução do paciente sem a supervisão de um profissional de nível superior. Quando o paciente era encaminhado para o atendimento médico, seguia para a cidade mais próxima muitas vezes, com o acompanhamento do profissional do posto -, cujo acesso, em termos geográficos, variava de quatro a vinte quilômetros. A dificuldade, no que diz respeito ao transporte do doente, estava sempre presente nos discursos apresentados, em especial, nas localidades de Irapuá, Missão, Três Soitas e Sede em Guarita, maior área indígena em extensão geográfica e população.

Ligeiro destacava-se por possuir apoio da prefeitura de Charrua em termos de transporte e, principalmente, de atendimento médico (Hökerberg, 1997). Apesar disso, Charrua possuía apenas um centro de saúde. Os casos mais graves eram encaminhados a Tapejara, onde existia um hospital de pequeno porte (menos de 100 leitos para internação). A área indígena de Carreteiro também utilizava Tapejara como referência para atendimento médico.

O Município de Rodeio Bonito servia de referência para a área indígena de Rio da Várzea; Iraí, para a área indígena de mesmo nome; e, por fim, os municípios de Redentora e Tenente Portela serviam de referência para a área indígena Guarita.

Todos os municípios utilizados como referência para atenção à saúde contavam com, pelo menos, um centro de saúde. Os hospitais tidos como referência local eram, de maneira geral, de pequeno porte (25 a 100 leitos) e conveniados ao SUS (Sistema Único de Saúde), dispondo de atendimento em clínica médica, pediatria, pré-natal, partos e cirurgia geral (Figura 2).

Em praticamente todas as áreas indígenas existiam relatos de pagamento de consultas médicas, mesmo quando aconteciam nos hos- pitais conveniados ao SUS. Ligeiro e Guarita (apenas nas localidades de Missão e Irapuá) recebiam apoio das prefeituras de Charrua e Redentora, respectivamente, no pagamento das consultas.

Quando era necessário atendimento em outras especialidades médicas, o paciente era encaminhado diretamente para as cidades de maior porte, como Erexim, Ijuí, Palmeiras das Missões, Frederico Westphalen e Passo Fundo. As sedes das DRS repassavam vacinas e medicamentos, além de funcionarem como unidades de referência para os programas de saúde (Figura 2).

O Município de Passo Fundo servia como referência à Região Norte do Estado, para onde drenavam os atendimentos de maior complexidade. A sede da ADR/FUNAI/RS funcionava como intermediária para a obtenção de internações ou mesmo de consultas nas especialidades médicas que não existiam ao nível local. O município possuía um Hospital Municipal, o único hospital público da região, além do Hospital São Vicente de Paulo e do Hospital da Cidade, estes últimos conveniados ao SUS.

Os moradores de Iraí, Inhacorá e Rio da Várzea utilizavam, em primeira instância, hospitais dos Municípios de Palmeiras das Missões e de Frederico Westphalen; em última análise, os de Passo Fundo devido à facilidade de acesso geográfico.

Nonoai, segunda maior área indígena em extensão geográfica e população, recorria aos hospitais locais de Nonoai e Planalto, no Estado do Rio Grande do Sul. Porém, os atendimentos de maior nível de complexidade eram efetuados no Município de Chapecó, Estado de Santa Catarina, tanto pela facilidade de acesso geográfico quanto pelo fato de esta área estar subordinada à ADR da FUNAI/SC, em Chapecó. Isto, entretanto, gerava conflitos interestaduais no repasse das Autorizações de Internação Hospitalar (AIHs) para as internações de indígenas.

Em última instância eram utilizados os serviços de saúde de Porto Alegre. Apesar da presença da ESAI/FUNASA, não existia qualquer ponto de apoio para os índios em trânsito. A única Casa do Índio existente na Região Sul do país situava-se em Curitiba e servia como ponto de apoio para pacientes necessitados de cuidados que demandavam tempo de permanência mais prolongado, como tratamentos radioterápicos e cirurgias corretivas para lábio leporino e fenda palatina.

A definição da natureza institucional dos hospitais incitava dúvidas. Não estava claro, para os profissionais que atuavam nas áreas in- 


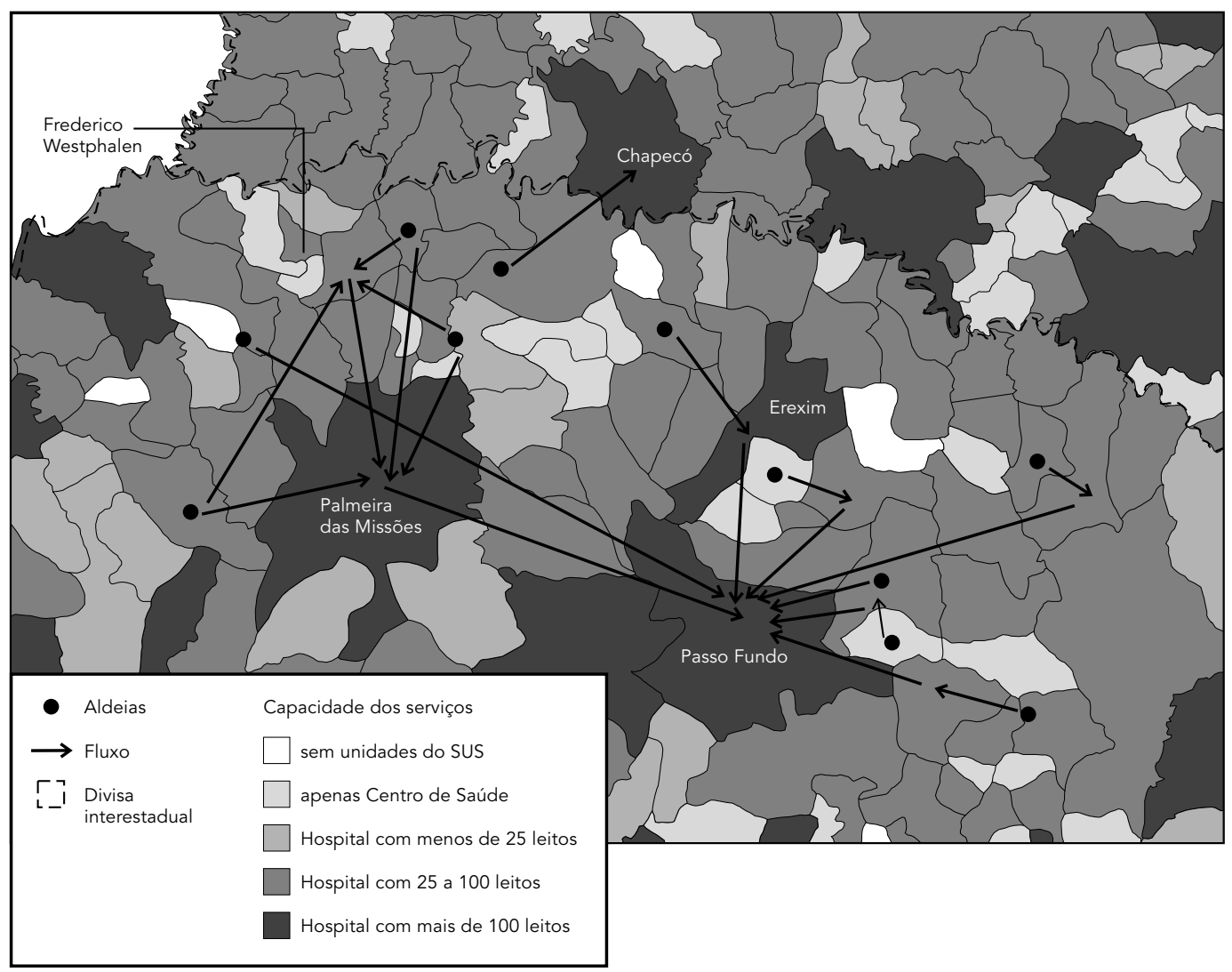

dígenas, se determinada instituição era pública, privada, conveniada ao SUS, filantrópica, bem como qual era o significado disso. A capacidade de resolução dos problemas de saúde era limitada tanto pelo difícil acesso aos recursos diagnósticos quanto pela dificuldade de comunicação dos pacientes indígenas com as instituições de saúde. As prefeituras locais, quando apoiavam as atividades em saúde das áreas indígenas, o que ficou melhor evidenciado em Redentora e Charrua, contribuíam com o atendimento médico de rotina e de urgência, com o transporte e com a distribuição de medicamentos.

A Figura 2 resume o caminho percorrido pelos indígenas em busca de assistência médica. O primeiro nível de atenção estava representado pelos municípios vizinhos às áreas indígenas. O segundo nível ficava no município de Frederico Westphalen, para onde se dirigiam os pacientes das áreas indígenas de Inhacorá, Iraí e Rio da Várzea, servindo como pólo intermediário entre essas áreas e a cidade de
Palmeiras das Missões. Também, o Município de Erexim poderia ser considerado como segundo nível de atenção à saúde, pois recebia os pacientes oriundos de Votouro. O terceiro nível de atenção estava representado por Passo Fundo e Chapecó, o último servindo de referência somente para os pacientes de Nonoai.

\section{- Os programas de saúde nas áreas indígenas}

O programa de controle de tuberculose é unificado e tem funções definidas nos níveis nacional, regional e distrital, subordinando-se a uma disciplina de programação com padrões técnicos e assistenciais bem definidos, além de garantir a distribuição gratuita de medicamentos e outros insumos necessários à assistência médica, o que deveria permitir o acesso universal da população às suas ações. Apesar de já ser amplamente divulgado no país, assim como de Noel Nutels ter implantado as ações voltadas para o controle da doença nas áreas indígenas 
da região norte e centro-oeste na década de cinqüenta (Costa, 1987), os profissionais das áreas indígenas Kaingáng do Rio Grande do Sul vinham atuando sem supervisão e reciclagem. Vale ressaltar que a tuberculose tem-se apresentado como problema crescente - em particular, após o aparecimento da Síndrome de Imunodeficiência Adquirida (AIDS) -, o que tornou ainda mais complexo o manejo dos pacientes, por exemplo, com a inclusão do aconselhamento para teste anti-HIV na rotina do atendimento aos doentes.

Quanto ao atendimento às gestantes, a maioria dos partos das índias Kaingáng ocorria em ambiente hospitalar. Os partos só foram realizados no domicílio quando não houve tempo hábil para o transporte das parturientes ao hospital (Hökerberg, 1997). Porém, a baixa freqüência de consultas durante o pré-natal mostrou-se evidente (Horta \& Cesar, 1996). Considerando que os relatos partiram apenas de profissionais de saúde, se é verdade que as mulheres Kaingáng no Rio Grande do Sul optaram pelo parto hospitalar, isto não resultou na melhoria na saúde tanto das parturientes quanto dos recém-natos, o que se traduziu no alto percentual de mortes perinatais $(16,8 \%)$, sem contar com o subregistro de óbitos por estas causas. O fato de estas mulheres não terem aderido ao acompanhamento pré-natal pode estar relacionado a conflito cultural entre elas e os profissionais de saúde, dificultando o acesso aos serviços de saúde. Uma alternativa seria a identificação de pessoas-chave, entre os agentes de saúde e/ou parteiras, para treinamento no que se refere ao acompanhamento pré-natal.

Quanto à prevenção do câncer de colo uterino, não existia programação clara nas áreas indígenas por parte da FUNAI ou da FUNASA, mas apenas medidas pontuais e isoladas por incentivo, principalmente, dos profissionais que atuavam nas áreas indígenas. Além de não haver programa de prevenção ao câncer de colo uterino implementado nas áreas indígenas, as mulheres que procuravam realizar o exame - a maioria com encaminhamento médico enfrentavam inúmeros obstáculos, entre os quais a distância, a necessidade de pagamento do exame, a demora para o retorno do resultado e a dificuldade na comunicação com o profissional de saúde - em particular, no que se refere à compreensão do significado do exame.

\section{A qualidade da assistência à saúde nas áreas indígenas Kaingáng: a utilização do Sistema de Informação Geográfica (SIG)}

Um dos aspectos essenciais para avaliar a qualidade da assistência à saúde é o acesso geográfico. A distância entre as áreas indígenas e a ADR da FUNAI, em Passo Fundo, era de 70 a $248 \mathrm{~km}$; as mais próximas eram Carreteiro e Ligeiro, e as mais distantes, Rio da Várzea, Votouro e Inhacorá. $\mathrm{O}$ acesso às áreas indígenas era feito por estradas pavimentadas em todo o percurso em Guarita, Cacique Doble, Iraí e Nonoai, e por não pavimentadas em parte do percurso, cerca de 12 a $80 \mathrm{~km}$ de distância, em Carreteiro e Votouro, respectivamente, além de Inhacorá, Ligeiro e Rio da Várzea, com trechos que variavam entre 20 e $30 \mathrm{~km}$.

Quanto ao perfil de mortalidade nas aldeias Kaingáng para o período de 1985-1995, observou-se que as doenças respiratórias foram responsáveis pela maioria dos óbitos indígenas $(19,7 \%)$, incluindo Votouro $(21,4 \%)$, Guarita (20,2\%), Inhacorá $(27,4 \%)$ e Iraí $(36,8 \%)$. Em Rio da Várzea, as doenças respiratórias empataram com as causas perinatais $(21 \%)$. Apesar de os sinais e sintomas mal definidos ocuparem a segunda posição no consolidado geral do período, correspondendo a $15,7 \%$ dos óbitos indígenas, quando se examinou cada área em separado, notou-se que, em Cacique Doble, esse grupo de causas foi responsável pela maioria dos óbitos $(38,6 \%)$, ao passo que, nas demais áreas, perdeu para as causas nutricionais e perinatais. As doenças relacionadas à nutrição ocuparam o terceiro lugar no consolidado geral dos óbitos $(15,4 \%)$, mas em Ligeiro foram responsáveis por $17,6 \%$ dos óbitos, pelo que foi a causa de óbito mais importante, seguida pelas causas perinatais $(16,7 \%)$. Estas também ocuparam o segundo lugar em Guarita (16,9\%), em Cacique Doble (12,3\%), onde dividiram a posição com as doenças infecciosas e parasitárias, e em Rio da Várzea (21,0\%), enquanto que, no consolidado geral, ocuparam a quarta posição $(14,1 \%)$.

Nas DRS que englobavam as áreas indígenas, as doenças cardiovasculares foram as causas predominantes de morte no ano de 1991 $(34,9 \%)$, seguidas pelas neoplasias $(17,9 \%)$, padrão completamente distinto das áreas indígenas, o que se justificava pela maior participação de idosos na composição etária da população do Estado do Rio Grande do Sul. Além disso, a falta de assistência médica podia ser um diferencial para a população indígena, o que pôde ser avaliado pela grande proporção de 
óbitos por causas mal definidas, representando $17,1 \%$ do total dos óbitos indígenas para o período entre 1985 e 1995, enquanto que para a sociedade envolvente correspondeu a apenas 7,1\% em 1991.

Para selecionar as categorias de óbitos que poderiam ser evitadas por medidas de atenção primária - como saneamento, imunização e assistência básica - ou através de rotinas preconizadas em programas financiados pelo Ministério da Saúde - o que é o caso do acompanhamento do crescimento e desenvolvimento de crianças, das atividades preconizadas para a atenção às infecções respiratórias e às diarréias, do programa de controle de tuberculose, entre outras -, adotamos como base a proporção de óbitos evitáveis por assistência primária de saúde em relação ao total dos óbitos ocorridos, dentre os quais, os sinais e sintomas mal definidos, a desnutrição, as pneumonias, as causas perinatais, a tuberculose, as causas maternas e o câncer de colo de útero (Tabela 1). Esta mesma classificação foi empregada na comparação com os dados de óbitos do Estado do Rio Grande do Sul, tendo como unidade de análise as DRS (Figura 3).

Nas áreas indígenas de Cacique Doble, Rio da Várzea e Guarita, os óbitos evitáveis representavam a maior parte das causas de óbitos, seguidos por Votouro e Ligeiro. Vale lembrar que este grupo de causas só não representou a maioria em Iraí e Carreteiro.

Em Inhacorá, Guarita e Rio da Várzea os óbitos evitáveis estavam representados tanto pelas causas mal definidas quanto pelas pneumonias e desnutrição, além das causas perinatais. Este padrão se repetiu, em menor escala, em Ligeiro e Votouro. Em Iraí, a desnutrição competiu com as pneumonias. Em Carreteiro, não houve sequer um caso de morte por desnutrição, fato provavelmente relacionado ao subregistro de óbitos, estando os óbitos evitáveis representados pelas causas perinatais, seguidas das pneumonias e das causas mal definidas.

Apesar de representarem parcela muito pequena do total dos óbitos indígenas ocorridos entre 1985 e 1995, os seis óbitos por tuberculose e os quatro óbitos por câncer de colo uterino correspondem a "eventos-sentinela" da má qualidade da assistência em saúde. A tuberculose provocou cinco óbitos em Guarita e um em Cacique Doble, enquanto o câncer de colo uterino provocou um óbito em Ligeiro, outro em Iraí e dois óbitos em Guarita.

Entre as doenças transmitidas sexualmente, foi encontrado um óbito por sífilis congênita em Iraí. Entre as doenças imunopreveníveis, o sarampo ocasionou dois óbitos na área indígena Guarita. As causas maternas foram responsáveis por um óbito em Ligeiro, outro em Cacique Doble e dois em Guarita, o que poderia ter sido prevenido mediante o acompanhamento pré-natal, o que não está distante da realidade das mulheres Kaingáng, que já buscavam os hospitais para terem seus filhos. Entre as parasitoses intestinais, a ascaridíase foi responsável por dois óbitos na aldeia Guarita. Da mesma forma, este conjunto de causas de óbitos ocorridos nas áreas indígenas em dez anos (1985-1995) representaram "eventos-sentinela” da baixa qualidade de assistência à saúde prestada aos índios Kaingáng no Rio Grande do Sul.

Basicamente, o que diferenciou as áreas indígenas foi a grande ocorrência de óbitos sem assistência médica, representados pelos sinais e sintomas mal definidos. A grande quantidade de óbitos por desnutrição poderia estar associada ao arrendamento das terras para colonos da região, em razão de restringir o espaço para plantio de subsistência em Ligeiro, Cacique Doble e Guarita.

Ao analisarmos a ocorrência de óbitos evitáveis no conjunto da população que forma a

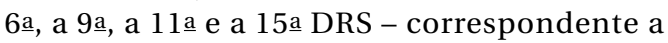
cerca de 20 a $30 \%$ do total dos óbitos, percentual bem menor do que o encontrado nas áreas indígenas - observamos que esteve representada principalmente pelo grupo de causas mal definidas.

Ou seja, os índios Kaingáng do Rio Grande do Sul estão geograficamente próximos de locais com certa capacidade física e diagnóstica, o que chega a ser exceção se pensarmos nas outras etnias espalhadas pelo país - em especial, na região amazônica - e, mesmo assim, a maioria dos óbitos ocorridos no período teria sido evitada por ações básicas de prevenção e atenção à saúde. Os serviços de saúde caracterizavam-se pelas dificuldades de relacionamento entre os índios e os profissionais de saúde, o que poderia explicar o fato de os indicadores dos índios apontarem para uma situação pior do que a encontrada entre os não-índios, com o que também se deparou Young (1988) em seu estudo a respeito dos índios no Canadá.

\section{Considerações finais}

As más condições de vida a que estavam submetidos os Kaingáng refletiram-se no quadro epidemiológico, em que se destacou a participação de óbitos por desnutrição, entre outras causas evitáveis por atenção primária à saúde, 


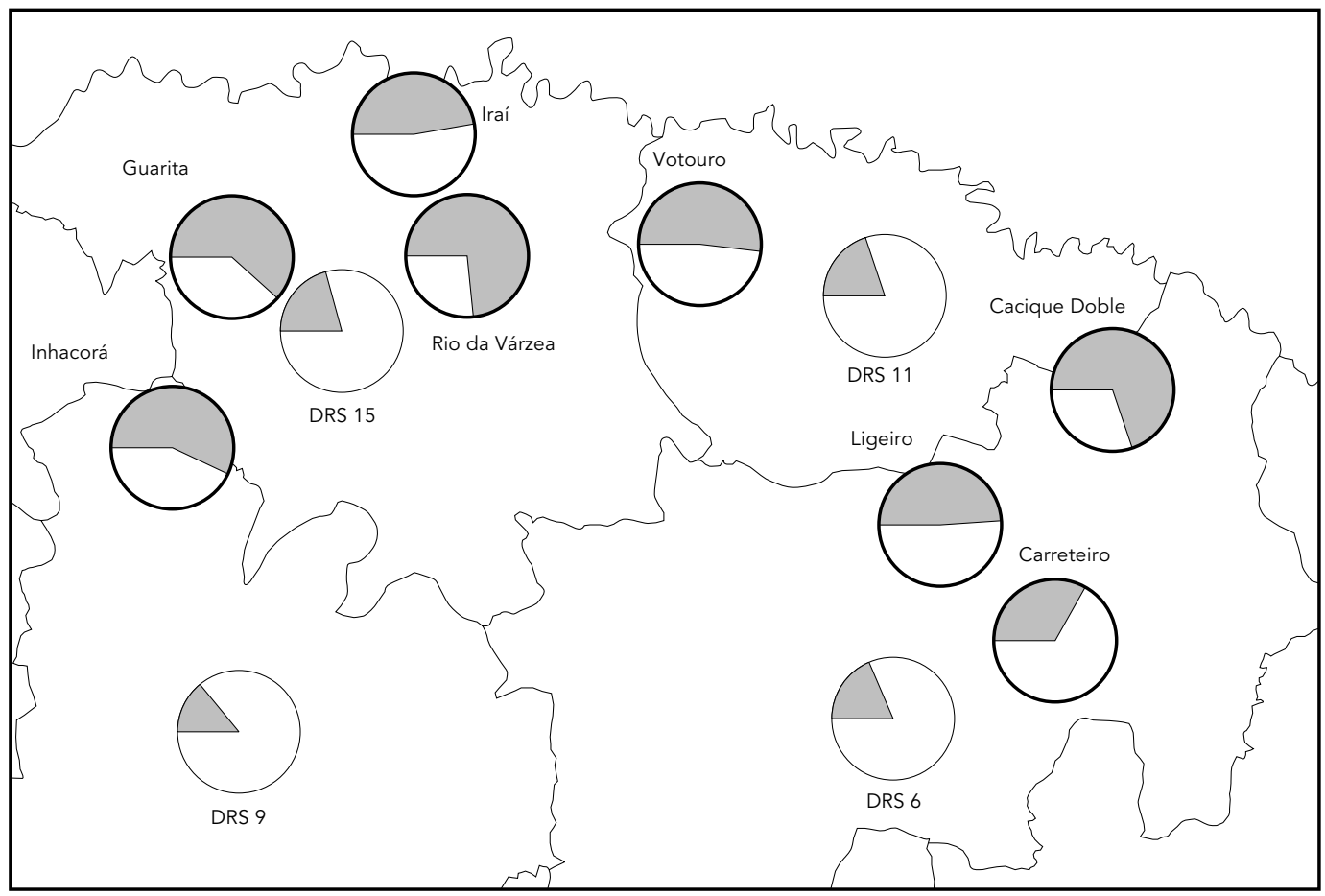

Proporção de óbitos

causas evitáveis $\bigcirc$ aldeias

demais causas $\bigcirc$ DRS

DRS: Delegacias Regionais de Saúde.

situação essa diferente da estrutura de mortalidade por causas da sociedade envolvente no Estado do Rio Grande do Sul.

A falta de organização dos serviços de saúde para o atendimento ao índio no Rio Grande do Sul era marcada pela dualidade institucional FUNAI e FUNASA, assim como pela escassez de recursos humanos que contemplassem as visões da saúde pública e a da antropologia. $\mathrm{O}$ amadurecimento nas relações entre as instituições envolvidas no atendimento à saúde do índio e a implantação do Distrito Sanitário Especial Indígena (DSEI) no Estado do Rio Grande do Sul possibilitaria a articulação com o SÚS, aproximando a lógica de funcionamento dos serviços de saúde existentes à realidade indígena local.

A intensificação das discussões a respeito da implantação de um sistema de informação para a saúde indígena é urgente, de modo a melhorar a qualidade em termos demográficos e epidemiológicos e possibilitar a análise comparativa da situação de cada grupo, assim como a avaliação das ações implementadas. $\mathrm{O}$ uso de SIG pode ser de grande utilidade para a implementação da idéia de sistema de informação, já que permite o acúmulo de grande número de informações, além de tornar possível a adequação dos indicadores à realidade que se pretende estudar. Em março de 2000, a FUNASA criou o Sistema de Informações da Atenção à Saúde Indígena (SIASI) no Seminário Nacional de Instituições de Ensino, Pesquisa e Assessoria Técnica, cujo primeiro módulo, o Cadastro da Família Indígena (CAFI) já está em fase de implantação (FUNASA, 2000a).

Os programas de controle da tuberculose, de prevenção ao câncer de colo uterino e o prénatal necessitam estar adequados à realidade cultural, não sendo resolutos na medida em 
que apenas reproduzem as diretrizes estabelecidas no nível nacional.

Quanto à área indígena de Nonoai, do ponto de vista da FUNASA, seria responsabilidade da Coordenação Regional do Rio Grande do Sul, ao passo que, para a FUNAI, seria da competência da Administração Regional de Chapecó, em Santa Catarina, com outra lógica de funcionamento, muito em virtude da falta de padronização das informações coletadas. Esse conflito administrativo ficou evidente, pois os índios atravessavam a fronteira estadual para buscar serviços de saúde em Chapecó, no Estado de Santa Catarina, apesar de essa área situar-se no Estado do Rio Grande do Sul.

Atualmente, o Subsistema de Atenção à Saúde Indígena está organizado na forma de 34 Distritos Sanitários Especiais Indígenas (DSEI) com definições de atribuições para os PólosBase, para as Casas de Saúde do Índio e para os Agentes de Saúde Indígena. Foi criado o DSEI Interior Sul, cuja sede fica em Chapecó, Estado de Santa Catarina, com Pólos-Bases em São Paulo, com sede em Bauru; Paraná, nas cidades de Guarapuava e Londrina; Santa Catarina e Rio Grande do Sul, com sede em Passo Fundo. Até o momento, a solução encontrada foi a articulação com municípios da região, os quais contrataram recursos humanos. Foram estabelecidas referências hospitalares em Passo Fundo, e os casos de maior complexidade devem ser encaminhados aos hospitais universitários das capitais dos Estados (FUNASA, 2000b). Porém, como foi discutido neste estudo, já existem referências preestabelecidas para atenção secundária e terciária em municípios de médio porte do Rio Grande do Sul, que poderiam ser oficializadas - como em Frederico Westhphalen, Erexim e Palmeiras das Missões - e melhor estabelecidas as parcerias com os hospitais universitários de Porto Alegre, na capital do Estado.

Finalmente, a metodologia empregada no presente estudo - em que se fez uso de todos os dados disponíveis, tomando em conta suas limitações - pode funcionar como ferramenta importante para o planejamento de ações de saúde em outras áreas indígenas brasileiras.

\section{Agradecimentos}

À Fundação Nacional de Saúde, que financiou as viagens para a pesquisa de campo, à Fundação Nacional do Índio, pelo apoio prestado durante essas viagens, ao professor Dr. Ulisses Eugenio Cavalcante Confalonieri, aos índios Kaingáng do Rio Grande do Sul e aos pareceristas dos Cadernos de Saúde Pública pelas valiosas contribuições. 


\section{Referências}

BARCELLOS, C. \& BASTOS, F. I., 1996. Geoprocessamento, ambiente e saúde: Uma união possível? Cadernos de Saúde Pública, 12:389-397.

CASTELLANOS, P. L., 1990. Avances metodológicos en epidemiologia. In: 1o Congresso Brasileiro de Epidemiologia, Anais, pp. 201-216. Campinas: ABRASCO/Universidade Estadual de Campinas.

CONFALONIERI, U. E. C., 1993. Perfis Epidemiológicos. In: Saúde de Populações Indígenas - Uma Introdução para Profissionais de Saúde (U. E. C. Confalonieri, C. B. Verani, D. P. Marinho \& D. D. L. Rigonatto, org.), pp. 15-26. Rio de Janeiro: Escola Nacional de Saúde Pública, Fundação Oswaldo Cruz.

COSTA, D. C., 1987. Políticas indigenistas e assistência à saúde. Noel Nutels e o Serviço de Unidades Sanitárias Aéreas. Cadernos de Saúde Pública, 3:388-401.

DE PAULA, S., 1991. Morrendo à Toa: Causas de Mortalidade no Brasil. São Paulo: Editora Ática.

DONABEDIAN, A., 1966. Evaluating the quality of medical care. Milbank Memorial Fund Quarterly, 44:166-203.

FUNASA (Fundação Nacional de Saúde), 2000a. FUNASA Cria Sistema Inédito de Informações sobre Saúde Indígena. 18 de agosto de 2000. http:// www.funasa.gov.br/not/notabr00/not57.htm>.

FUNASA (Fundação Nacional de Saúde), 2000b. Atenção à Saúde dos Povos Indígenas: Caracterização e Estágio da Implantação dos Distritos Sanitários Especiais Indígenas. 18 de agosto de 2000. <http:// www.funasa.gov.br/ind/ind01.htm>.

HÖKERBERG,Y. H. M., 1997. A Situação de Saúde dos Índios Kaingáng do Rio Grande do Sul, 1985-1995. Dissertação de Mestrado, Rio de Janeiro: Escola Nacional de Saúde Pública, Fundação Oswaldo Cruz.

HORTA, B. L. \& CESAR, J. A., 1996. Diagnóstico de Saúde da População Indígena. Rio Grande do Sul. Porto Alegre: Coordenação Regional da Fundação Nacional de Saúde do Rio Grande do Sul. (mimeo.)

IBGE (Fundação Instituto Brasileiro de Geografia e Estatística), 1992. Pesquisa Nacional sobre Assistência Médico-sanitária. Rio de Janeiro: IBGE.
IBGE (Fundação Instituto Brasileiro de Geografia e Estatística), 1997. Malha Municipal do Brasil. CD-ROM. Rio de Janeiro: IBGE.

KUNITZ, S., 1983. Disease patterns on the Navajo Reservation. In: Disease Change and the Role Medicine: The navajo experience (S. Kunitz, ed.), pp. 64-117, Berkeley: University of California Press.

LOBO, M. S., 1996. O Caso Yanomámi do Brasil: Uma Proposta Estratégica de Vigilância Epidemiológica. Dissertação de Mestrado, Rio de Janeiro: Escola Nacional de Saúde Pública.

MINAYO, M. C., 1993. O Desafio do Conhecimento: Pesquisa Qualitativa em Saúde. São Paulo: Editora Hucitec/Rio de Janeiro: ABRASCO.

MS (Ministério da Saúde), 1997. Sistema de Informação sobre Mortalidade. CD-ROM. Brasília: Centro Nacional de Epidemiologia/Departamento de Informática do SUS.

OPS (Organización Panamericana de la Salud), 1988. Investigación sobre perfiles de salud. Antecedentes de los proyectos sobre análisis de mortalidad. Boletin Epidemiologico, 9:1-3.

OPS (Organización Panamericana de la Salud), 1996. Uso de los Sistemas de Información Geográfica en Epidemiología (SIG-Epi). Boletin Epidemiologico, 17:1-6.

RUTSTEIN, D. D., 1976. Measuring the quality of medical care. A clinical method. New England Journal of Medicine, 294:582-588.

SSMA (Secretaria de Saúde e Meio Ambiente), 1993. A Criança e o Adolescente no Rio Grande do Sul: Indicadores de Saúde. Rio Grande do Sul: Secretaria de Saúde e Meio Ambiente.

VIACAVA, F. \& BAHIA, L., 1996. Assistência médicosanitária. Os serviços de saúde segundo o IBGE. Dados-Radis, 20.

WHITE, F., 1998. De la evidencia al desempeño: Cómo fijar prioridades y tomar buenas decisiones. Revista Panamericana de Salud Publica, 4:69-74.

WIRSING, R., 1985. The health of traditional societies and the effects of acculturation. Current Anthropology, 26:303-322.

YOUNG, T. K., 1988. Are subartic indians undergoing to epidemiologic transition? Social Science and Medicine, 26: 659-71. 